\title{
The high prevalence of inappropriate feeding among infants presenting with an apparent life-threatening event
}

\author{
Katsuaki Kojima ${ }^{1}$, Katie Mckinley $^{2}$, Pamela Donohue ${ }^{3}$, Yakov Sigal ${ }^{1}$ \\ ${ }^{1}$ Department of Pediatrics and Human Development, College of Human Medicine, Michigan State University, East \\ Lansing, Michigan, ${ }^{2}$ Maternal Child Health Division, Ingham County Health Department, Lansing, Michigan, ${ }^{3}$ Division \\ of Neonatology, Department of Pediatrics, Johns Hopkins University School of Medicine, Baltimore, Maryland. \\ E-mail: fi990071@gmail.com
}

Received: 30th January 2017, Revised: 19th March 2017, 20th March 2017

SUMMARY: Kojima K, Mckinley K, Donohue P, Sigal Y. The high prevalence of inappropriate feeding among infants presenting with an apparent lifethreatening event. Turk J Pediatr 2017; 59: 304-310.

\begin{abstract}
Although there are anecdotal reports of a link between inappropriate feeding and an apparent life-threatening event (ALTE), previous studies have not examined this association in a cohort of affected infants. This study compared the feeding behaviors of infants who have had an ALTE with age- and sexmatched controls. This is a single-center case control study. Forty-six term infants aged 6 months or less, who were hospitalized over a 34-month period following an ALTE, comprised the study sample; 92 age- and sex-matched controls were recruited from a wellness clinic. Feeding practices reported by parents were evaluated for their appropriateness with respect to the volume of each feeding, and the frequency and total volume of feedings per 24-hour period, based on the American Academy of Pediatrics guidelines. Comparisons were made between the ALTE cases and controls. Inappropriately fed infants were compared with the rest of the sample. The ALTE and control groups were similar with respect to the prevalence of breastfeeding, insurance, birth weight, and weight percentile at presentation. The ALTE group had a lower prevalence of appropriate feeding compared to the control group ( $43.5 \%$ versus $63.0 \%, p=0.029)$. Overfed infants were at a higher weight percentile at the time of presentation $\left(46.5^{\text {th }}\right.$ percentile versus $31.4^{\text {th }}$ percentile, $\mathrm{p}=0.037$ ). These results represent the association between ALTE and inappropriate feeding practice, which emphasizes the need for assessment and education regarding feeding practices in patient presenting with an ALTE.
\end{abstract}

Key words: overfeeding, breastfeeding, formula, apparent life threatening event.

An apparent life threatening-event (ALTE) is defined as a caretaker-observed frightening episode, characterized by apnea, color change, change in muscle tone, and choking, or gagging ${ }^{1}$. The incidence of ALTEs ranges from 0.5-3 per 1,000 live births ${ }^{2,3}$. Gastroesophageal reflux disease (GERD), seizures, and lower respiratory tract infections are the most common diagnoses in infants presenting with an ALTE; however, many cases are idiopathic ${ }^{4}$. Although inappropriate feeding has been suggested as a risk factor for experiencing an ALTE, only anecdotal reports have been published ${ }^{5}$. Difficulty defining appropriate feeding and ascertaining actual feeding practices in the home are methodological issues that have impeded research in this area.

Despite these challenges, we decided to explore the association between ALTEs and inappropriate feeding practices, as they can be modified through parental education at a minimal cost. This study tested the hypothesis that ALTEs are associated with inappropriate volume or frequency of feedings as per the American Academy of Pediatrics (AAP) guidelines ${ }^{6}$.

\section{Material and Methods}

\section{Study design}

This was a single-center case control study. The study was exempted from the human subjects 
review by the institutional review boards of Michigan State University, Sparrow Hospital, and Ingham County Health Department.

\section{Population}

Sparrow Hospital is the largest hospital system in the mid-Michigan area. Its pediatric inpatient units admit over 1,500 patients annually. Infants who were less than 6 months of age who admitted to the pediatric floor between January 1st, 2012 and November 1st, 2014 with a diagnosis of ALTE were included in the study. We used the ICD-9 code, 799.82 to identify ALTE cases. Those who were born preterm (less than 37 weeks gestational age), and those known to have a feeding difficulty were excluded. In cases of multiple hospitalizations during the study period, only the first admission was included. For each case, age- and sex-matched controls were selected from those who visited the Well Child Health Center for routine health care.

The following information was retrieved from the medical records of both ALTE cases and controls: chronological age (in days), sex, insurance (commercial, Medicaid, or self), gestational age at birth, birth weight (in grams), weight at presentation (in grams), length at presentation (in centimeters), details of feeding (such as exclusive breast feeding, mixed or formula feeding), type of formula, volume per feeding (in ounces), and frequency of feedings (times per 24 hours). Feeding information is retrieved from medical records and is based on parental report.

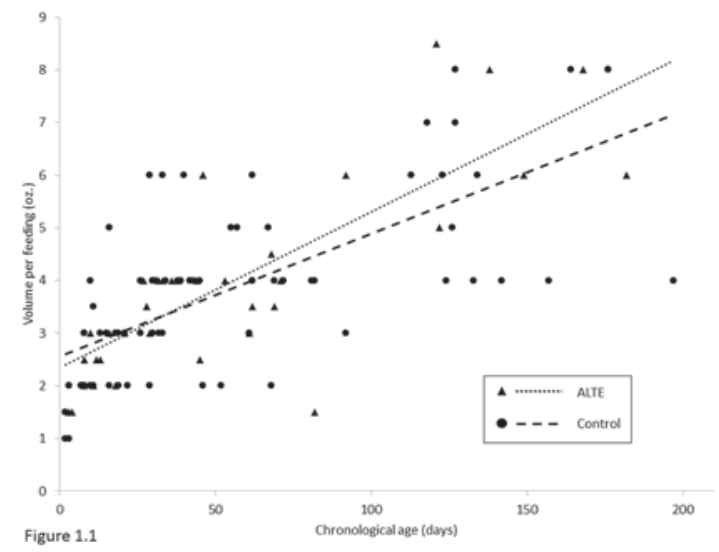

Fig. 1. Scatter plot of volume of each feeding and chronological age. Triangle and circle represent the ALTE group and the control group, respectively. Dotted regression line and dashed regression line represent the ALTE group and the control group, respectively.

\section{Operational definitions}

The appropriateness of the volume and frequency of feedings was determined based on the nutritional guidelines published by the American Academy of Pediatrics (AAP) ${ }^{6}$. The guidelines have age-specific recommendations for the volume and frequency of feedings for both formula-fed and breastfed infants (Tables I, II). However, these recommendations were made for specific ages, not age ranges. Hence, if an infant's age was between those indicated in the Table, we combined the parameter ranges of the ages above and below to create a broader range (e.g., the recommended amount per feeding for infants 1-month and 2-months old was 2 to $4 \mathrm{oz}$. and 4 to $5 \mathrm{oz}$. respectively;

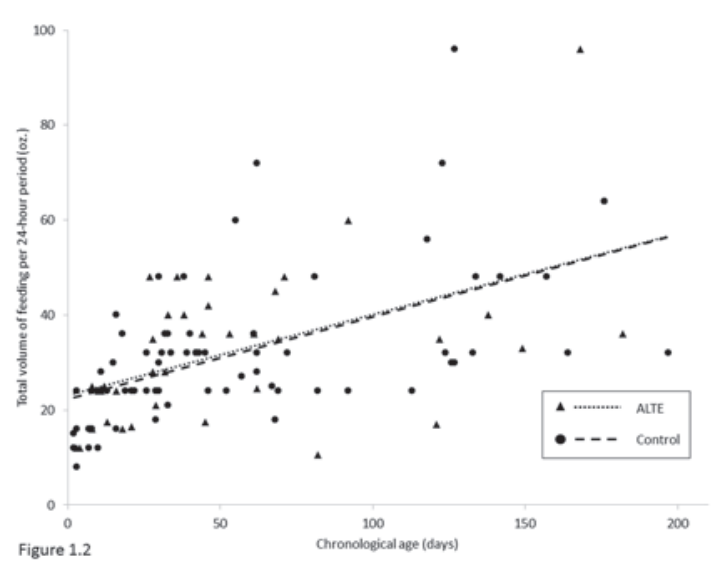

Fig. 2. Scatter plot of total volume of feeding per 24-hour period and chronological age. Triangle and circle represent the ALTE group and the control group, respectively. Dotted regression line and dashed regression line represent the ALTE group and the control group, respectively.

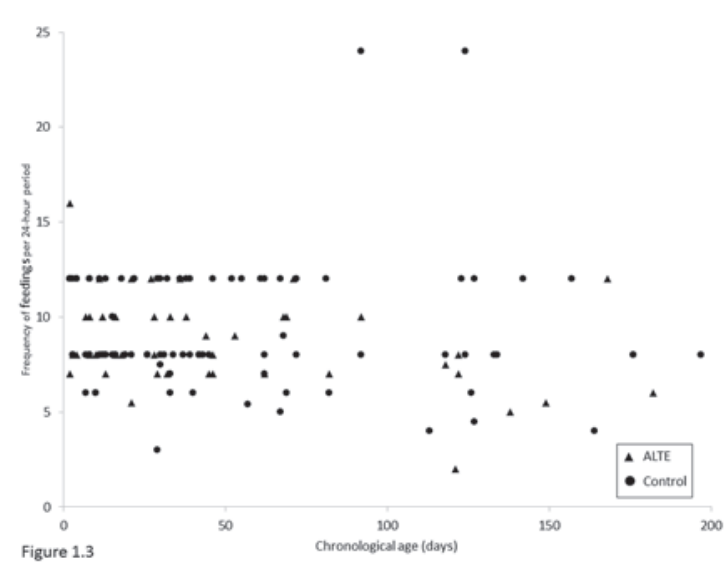

Fig. 3. Scatter plot of frequency of feeding per 24-hour period and chronological age. Triangle and circle represent the ALTE group and the control group, respectively. 
Table I. AAP Guidelines for Appropriate Feeding in Formula-fed Infants.

\begin{tabular}{llll}
\hline Age & Volume of each feeding (oz.) & $\begin{array}{l}\text { Frequency of feedings } \\
\text { per } 24 \text { hours }\end{array}$ & $\begin{array}{l}\text { Total volume of feedings } \\
\text { per 24 hours (oz.) }\end{array}$ \\
\hline 3 to 5 days & 2 & 8 to 12 & 20 \\
1 month & 2 to 4 & 6 to 8 & 20 to 31 \\
2 months & 4 to 5 & 6 to 8 & 21 to 32 \\
4 months & 4 to 6 & 6 to 8 & 26 to 36 \\
\hline
\end{tabular}

Table I-II. AAP Guidelines for Appropriate Feeding in Breastfed Infants.

\begin{tabular}{ll}
\hline Age & Frequency of feedings per 24 hours \\
\hline Initial week & 10 to 12 \\
2 months & 8 to 12 \\
After several months & 6 to 12 \\
\hline
\end{tabular}

American Academy of Pediatrics Bright Futures Nutrition, 3rd Edition. American Academy of Pediatrics; 2011.

hence, the appropriate volume per feeding for a 1.5 -month old was set at 2 to $5 \mathrm{oz}$.). The appropriate total volume of feedings per 24hour period for 3 to 5 -day olds was indicated as $20 \mathrm{oz}$. in the AAP guidelines. However, given the marked variations in the number of feedings per 24 hours, we converted the recommended $20 \mathrm{oz}$. to a range of 16 to 24 oz. per 24 hours. Less than 2 oz. per feeding was set as appropriate for infants less than 3 days old. The guidelines also did not indicate the appropriate volume per feeding or the total volume of feeding per 24 hours for breastfed infants since the World Health Organization recommends breastfeeding as much as the child wants. For the purpose of the current research, we used the parameters provided for the formula-fed infants of the same age to determine the appropriateness in such cases. The volume of feedings for breast-fed infants was determined using the volume of expressed breast milk given to the infants. We were unable to measure the volume of breast milk unless mothers expressed the milk. We did not use caloric density information, as we focused only on previously healthy term infants in whom concentrated formula was not warranted. Feeding was considered inappropriate if the volume of each feeding, or the frequency, or total volume of feedings over a 24-hour period was outside the set range. These criteria were applicable to exclusively breastfed and exclusively formula-fed infants. For infants who received a combination of breast-milk and formula, the frequency and volume of feedings were estimated as the combined frequency and volume of both types of feedings. Overfeeding is evident when the volume of each feeding, the frequency of feedings, or the total volume of feedings in a 24-hour period is above the recommended range. Inappropriate feeding also includes underfeeding, wherein the volume of each feeding, the frequency of feedings, or the total volume of feedings per 24 hours is below the recommended range. For example, if a 1-month old infant consumed $6 \mathrm{oz}$. of formula 12 times a day, it met the criteria for inappropriate overfeeding. Similarly, feeding that was below the set range was considered underfeeding.

\section{Data analysis}

Demographic and clinical characteristics were analyzed using descriptive statistics and compared between cases and controls. Proportions of inappropriately fed, overfed, and underfed infants were compared between ALTE cases and controls. The number and proportion of infants with appropriate volumes per feeding, frequencies of feedings per 24hour period, and total volume of feedings per 24 hours, were compared between the ALTE cases and controls. Weight and height percentiles at presentation were computed by sex- and age-specific clinical growth charts created by the Centers for Disease Control and Prevention ${ }^{7}$. Weight, percentile weight, height, and percentile height at presentation were compared between the cases and controls. 
Table II. Demographic and Clinical Characteristics of Apparent Life-threatening Event Cases and Controls.

\begin{tabular}{llll}
\hline & ALTE $(\mathrm{n}=46)$ & Control $(\mathrm{n}=92)$ & p value \\
\hline Age - median days (range) & $32.5(2-182)$ & $33(2-197)$ & 0.960 \\
Gender - male (proportion) & $23(50 \%)$ & $46(50 \%)$ & 1.000 \\
$\begin{array}{l}\text { Insurance - Medicaid (proportion) } \\
\text { Birth weight - median gram (range) }\end{array}$ & $24(52.2 \%)$ & $20(33.3 \%)$ & 0.123 \\
$\begin{array}{l}\text { Weight at presentation - median gram } \\
\text { (range) }\end{array}$ & $4260(2210-4290)$ & $3280(2320-4230)$ & 0.797 \\
$\begin{array}{l}\text { Percentile weight at presentation - median } \\
\text { percentile (range) }\end{array}$ & $33.8(0.9-87.9)$ & $34.6(1.3-97.2)$ & 0.873 \\
$\begin{array}{l}\text { Length at presentation - median centimeter } \\
\text { (range) }\end{array}$ & $54.0(44.5-66.0)$ & $53.3(42.5-68.6)$ & 0.444 \\
$\begin{array}{l}\text { Percentile length at presentation - median } \\
\text { percentile (range) }\end{array}$ & $51.9(0.3-99.2)$ & $27.8(0.1-83.7)$ & 0.020 \\
\hline
\end{tabular}

All overfed infants in the sample were compared with the rest of the sample. Birth weight and percentile weight at presentation were compared between infants who were overfed and the other infants.

We used the Fisher's exact test, chi-square test, and Mann-Whitney test for the appropriate statistical analyses. The analyses were performed using STATA version 14.1 for Windows 8 (StataCorp. 2015, Stata Statistical Software: Release 14, College Station, TX: StataCorp LP.).

\section{Results}

During the study period, there were 70 ALTE admissions to the pediatric unit; 24 were excluded from the study. Seventeen infants were born prematurely, 2 had a known history of feeding difficulty ( 1 had a known genetic condition and 1 had laryngomalacia), 1 was older than 7 months of age, 3 were repeat admissions, and 1 did not have the requisite information in the medical record. For the 46 cases that were included in the study, 92 age- and sex-matched controls were identified (Table II).

When compared to the control group, the ALTE group had a significantly lower proportion of appropriate feedings $(43.5 \%$ vs $63.0 \%, \mathrm{p}=$ 0.029) (Table III). There were no significant differences between the ALTE group and the control group with respect to proportions of overfeeding or underfeeding. In the ALTE group, $18(69.2 \%)$ out of 26 cases with inappropriate feedings were overfed compared to $24(70.6 \%)$ out of 34 in the control group $(\mathrm{p}=0.566)$. The volume per feeding, total volume of feedings per 24-hour period, and frequency of feedings per 24-hour period for the control group and the ALTE group are shown in Figures 1.1, 1.2 , and 1.3 respectively. Slopes of regression lines in Figure 1 represent the rate of change in volume per feeding for each chronological age. Slopes of regression lines did not differ between the control group and the ALTE group ( $p=0.403)$. Similarly, the slopes of regression lines in Figure 2 represent the rate of change in total volume of feeding per 24hour period for each chronological age. Slopes of regression lines did not differ between the control group and the ALTE group ( $p=0.734)$. The proportions of infants with appropriate volumes for each feeding, frequency of feedings, and total volume of feedings per 24 hours did not differ between the ALTE and control groups (Table III). Birth weight, weight at presentation, percentile weight at presentation, and length at presentation were similar for both groups. Percentile length at presentation was lower in the control group (Table II).

Of the 138 infants, 60 received inappropriate feedings; 23 (38\%) received inappropriate volumes during individual feedings, 40 (67\%) had inappropriate frequencies of feeding, and $45(75 \%)$ had inappropriate total volumes in feedings per 24-hour period. Twenty-one (35\%) of the 60 infants who received inappropriate 
Table III. Comparison of Feeding Practices between Apparent Life-threatening Event Cases and Controls.

\begin{tabular}{llll}
\hline & ALTE $(\mathrm{n}=46)$ & Control (n=92) & p value \\
\hline Exclusively breast fed - (proportion) & $13(28.3 \%)$ & $24(26.1 \%)$ & 0.019 \\
Exclusively formula fed - (proportion) & $28(60.9 \%)$ & $39(42.4 \%)$ & \\
Mixed feeding - (proportion) & $5(10.9 \%)$ & $29(31.5 \%)$ & \\
Cow's milk formula - (proportion) & $15(45.5 \%)$ & $47(69.1 \%)$ & 0.131 \\
Partially hydrolyzed formula - (proportion) & $10(30.3 \%)$ & $11(16.2 \%)$ & \\
Extensively hydrolyzed formula - (proportion) & $2(6.1 \%)$ & $3(4.4 \%)$ & \\
Amino acid formula - (proportion) & $2(6.1 \%)$ & $0(0 \%)$ & \\
Soy formula - (proportion) & $3(9.1 \%)$ & $5(7.4 \%)$ & 0.029 \\
Other formula - (proportion) & $1(3.0 \%)$ & $2(2.9 \%)$ & 0.116 \\
Appropriately fed - (proportion) & $20(43.5 \%)$ & $58(63.0 \%)$ & 0.295 \\
$\begin{array}{l}\text { Overfed - (proportion) } \\
\text { Underfed - (proportion) }\end{array}$ & $18(39.1 \%)$ & $24(26.1 \%)$ & 0.518 \\
$\begin{array}{l}\text { Volume of each feeding - appropriate } \\
\text { (proportion) }\end{array}$ & $8(17.4 \%)$ & $10(10.9 \%)$ & 0.063 \\
Frequency of feedings per 24 & $37(80.4 \%)$ & $78(84.8 \%)$ & \\
hours - appropriate (proportion) & $28(60.9 \%)$ & $70(76.1 \%)$ & 0.054 \\
$\begin{array}{l}\text { Total volume of feedings per 24 } \\
\text { hours - appropriate (proportion) }\end{array}$ & $26(56.5 \%)$ & $67(72.8 \%)$ & \\
\hline
\end{tabular}

feedings met one criterion of its definition, 30 (50\%) met two criteria, and 9 (15\%) met all three criteria; 42 (70\%) of them were overfed. The birth weight of the overfed infants and the other infants was similar (median: 3,320 grams versus 3,270 grams, $\mathrm{p}=0.755)$. However, the median weight percentile at presentation was significantly higher in the infants who were overfed $\left(46.5^{\text {th }}\right.$ percentile versus $31.4^{\text {th }}$ percentile $\mathrm{p}=0.037$ ).

\section{Discussion}

We found that ALTE group had a higher proportion of inappropriate feeding compared to control group. The infants who were inappropriately fed were more often overfed rather than underfed. The prevalence of overfeeding was similar in both the ALTE and control groups. Infants who were overfed had greater weights at presentation compared to the others in the sample.

To the best of our knowledge, no previous study has examined the association of inappropriate feeding with ALTEs in a cohort of infants.
Only anecdotal evidence of overfeeding as a risk factor for an ALTE, has been reported ${ }^{5}$, which might be due to reports of an association between overfeeding and GERD ${ }^{8}$. GERD has frequently been cited as a cause of ALTEs ${ }^{9}$, but a causal relationship has not been verified ${ }^{10}$. Although our study found an association between inappropriate feeding and ALTEs, it did not confirm any distinct associations between overfeeding or underfeeding and ALTEs. These findings suggest that the underlying mechanism through which inappropriate feeding predisposes an infant to an ALTE may not be an exacerbation of GERD, but rather a dysfunctional coordination between respiration and swallowing. A recent study suggested that ALTEs are associated with esophageal dysmotility rather than GERD ${ }^{11}$, which supports this hypothesis. Inappropriate feeding might also be an indicator of suboptimal childcare, which could be an alternative explanation for the occurrence of an ALTE. The existing literature suggesting an association between ALTEs and child abuse ${ }^{12,13}$, supports this hypothesis. However, a causal relationship 
between the quality of childcare and ALTEs remains unclear.

One of the strengths of our study is the use of established criteria to determine the appropriateness of feeding practices. The AAP's nutritional guidelines for appropriateness of volume and frequency of feeding are applicable to the general pediatric population in the US. We excluded preterm infants, as their body sizes are smaller relative to term infants of the same chronological age. We also verified that the ALTE and control groups had similar body sizes in relation to their chronological ages at the time of presentation using weight percentiles at presentation.

Although a causal relationship between ALTEs and inappropriate feeding cannot be proven by this retrospective study, we speculate that either ineffective esophageal motility or suboptimal childcare could account for the ALTEs in our study. Detecting inappropriate feeding practices and providing appropriate education to correct them, is likely to prevent the development of an ALTE produced by either scenario. Therefore, it is important that primary care physicians perform thorough feeding evaluations during routine physical examinations to detect at-risk infants and prevent ALTEs.

When information regarding feeding practices is based solely on parental reports, its reliability may be questionable. However, a study using parental reports found that a higher number of feedings per day predicted weight gain ${ }^{14}$. In addition, our own study, which used parental reports, also found that overfeeding was associated with higher weight percentiles at presentation; this finding supports the accuracy of the parental reports. In our study, the ALTE group included only patients admitted to the general pediatric ward, which might have resulted in the exclusion of patients who were not affected as severely, and therefore, did not require hospitalization. However, due to the subjective nature of an ALTE, it seemed reasonable to include only the more severely affected patients in the study. The information that was collected regarding feedings was limited to their volumes and frequencies at the time of the visit. We might have been able to delineate further the relationship of ALTEs with feeding practices if we had collected feeding information that was more detailed, such as the length and timing of the feedings in relation to other activities, recent changes in formula, and the temporal relationship of the ALTE with the last feeding. However, due to the retrospective nature of the study, such information was unavailable. Future prospective studies that include such detailed histories would be more informative. In our study, a final diagnosis could not be included due to limited access to medical records. However, the fact that our study found an association between ALTEs and inappropriate feeding, regardless of the final diagnosis, points to the robustness of our findings.

In conclusion, inappropriate feeding is associated with ALTEs based on our singlecenter case control study. Education regarding feeding practices is an essential part of general pediatric practice and our study emphasizes its importance. Multiple studies in the past have suggested that routine work-ups are unnecessary when evaluating a child presenting with an ALTE ${ }^{12,15}$. A detailed history and physical examination are the most important tools, as in other medical conditions. Our study affirms the importance of obtaining a detailed history, including feeding practices when evaluating a pediatric patient presenting to the emergency department with an ALTE.

\section{Acknowledgement}

This work was supported by GMEI mini grants awarded to K. Kojima.

\section{REFERENCES}

1. US Department of Health and Human Services Infantile apnea and home monitoring: report of a consensus development conference. Publication NIH 1987: 872905.

2. Kiechl-Kohlendorfer U, Hof D, Peglow UP, et al. Epidemiology of apparent life threatening events. Arch Dis Child 2005; 90: 297-300.

3. Semmekrot BA, Van Sleuwen BE, Engelberts AC, et al. Surveillance study of apparent life-threatening events (ALTE) in the Netherlands. Eur J Pediatr 2010; 169: 229-236.

4. McGovern MC, Smith MB. Causes of apparent life threatening events in infants: a systematic review. Arch Dis Child 2004; 89: 1043-1048.

5. Elzouki AY, Harfi HA, Stapleton F, et al. Textbook of clinical pediatrics. Springer Science \& Business Media; 2012.

6. American Academy of Pediatrics Bright Futures Nutrition, ( $3^{\text {rd }}$ ed). American Academy of Pediatrics; 2011. 
7. Centers for Disease Control and Prevention. Clinical Growth Charts. Retrieved on August 232015 from http://www.cdc.gov/growthcharts/clinical_charts.htm Published 8/23, 2015.

8. Orenstein SR, McGowan JD. Efficacy of conservative therapy as taught in the primary care setting for symptoms suggesting infant gastroesophageal reflux. J Pediatr 2008; 152: 310-14. e1.

9. Spitzer AR, Boyle JT, Tuchman DN, et al. Awake apnea associated with gastroesophageal reflux: a specific clinical syndrome. J Pediatr 1984; 104: 200-205.

10. Tolia V, Vandenplas Y. Systematic review: the extraoesophageal symptoms of gastro-oesophageal reflux disease in children. Aliment Pharmacol Ther 2009; 29: $258-272$.
11. Hasenstab KA, Jadcherla SR. Respiratory events in infants presenting with apparent life threatening events: is there an explanation from esophageal motility? J Pediatr 2014; 165: 250-55 el.

12. Tieder JS, Altman RL, Bonkowsky JL, et al. Management of apparent life-threatening events in infants: a systematic review. J Pediatr 2013; 163: 94-9 e1-6.

13. Bonkowsky JL, Guenther E, Filloux FM, et al. Death, child abuse, and adverse neurological outcome of infants after an apparent life-threatening event. Pediatrics 2008; 122: 125-131.

14. Worobey J, Pena J, Ramos I, et al. Infant difficulty and early weight gain: does fussing promote overfeeding? Matern Child Nutr 2014; 10: 295-303.

15. Brand DA, Altman RL, Purtill K, et al. Yield of diagnostic testing in infants who have had an apparent life-threatening event. Pediatrics 2005; 115: 885-893. 